Research Paper

\title{
CA724 Predicts Tumor Regression Grade in Locally Advanced Gastric Cancer Patients with Neoadjuvant Chemotherapy
}

Yilin Tong, MD ${ }^{1}$; Yanmei Zhu, MD, PhD²; Yan Zhao, MD, PhD ${ }^{1}$; Chengyao Jiang, MD ${ }^{1}$; Wentao Wang,

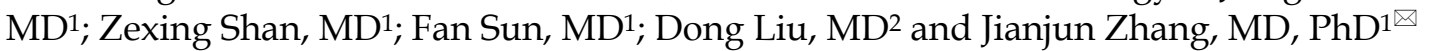

1. Department of Gastric Surgery, Liaoning Cancer Hospital and Institute, Cancer Hospital of China Medical University, Shenyang, China.

2. Department of Pathology, Liaoning Cancer Hospital and Institute, Cancer Hospital of China Medical University, Shenyang, China.

$\triangle$ Corresponding author: Jianjun Zhang, MD, PhD, Department of Stomach Surgery, Liaoning Cancer Hospital and Institute (Cancer Hospital of China Medical University), No 44 of Xiaoheyan Road, Dadong District, Shenyang 110042, China. Tel: 86-18900918910; Fax: 86-24-24315679; E-mail: zhangjianjun@cancerhosp-ln-cmu.com.

(c) The author(s). This is an open access article distributed under the terms of the Creative Commons Attribution License (https://creativecommons.org/licenses/by/4.0/). See http:/ /ivyspring.com/terms for full terms and conditions.

Received: 2021.03.21; Accepted: 2021.08.23; Published: 2021.09 .03

\begin{abstract}
Purpose: Tumor regression grade (TRG) is widely used to evaluate the efficacy of neoadjuvant chemotherapy (NCT) and it is related to many clinicopathological factors. However, whether TRG can be predicted by clinical characteristics is unknown.

Methods: 141 locally advanced gastric cancer (GC) patients who underwent NCT and curative operation were retrospectively analyzed. TRG is reevaluated according to the CAP guideline. The values of CA199, CA125 and CA724 before NCT (pre-) and after NCT (post-) were extracted from our database. Survival curves on overall survival (OS) were obtained by Kaplan-Meier method, and differences were analyzed by log-rank test. Associations between categorical variables were explored by chi-square test or Fisher's exact method. Univariable and multivariate analyses were performed by logistic regression model or Cox proportional hazard regression model.

Results: TRG was related to OS $(P<0.001)$, especially when divided into responders (TRG $0-1)$ and non-responders (TRG 2-3). Pre-CA724 $(p=0.029)$ and post-CA199 $(p=0.038)$ were related to OS. In multivariable analysis, pre-CA724 $(p=0.015)$ and post-CA199 $(p=0.007)$ were independent prognostic factors for OS, respectively. The changes (diff-) of all tumor markers were not related to OS. Among the clinical characteristics, pre-CA724 $(P=0.047)$ and tumor size $(P=0.012)$ were related to TRG, while pre-CA199 $(P=0.377)$ and pre-CA125 ( $P=0.856)$ were not. In logistics analysis, pre-CA724 $(P=0.032)$, tumor size $(P=0.011)$ and tumor location $(P=0.047)$ were independent risk factors to pathological response.

Conclusion: CA724 was an independent prognostic factor for OS and could be used to predict pathological response.
\end{abstract}

Key words: Gastric cancer; Tumor regression grade; Neoadjuvant therapy; Tumor marker; CA724

\section{Introduction}

Gastric cancer (GC) is a common digestive tract malignancy, and third leading cause of death worldwide [1]. Neoadjuvant chemotherapy (NCT) followed by surgery has become a recommended treatment for locally advanced gastric cancer, because the results of many clinical trials showed NCT could induce tumor down-staging [2], tumor volume reducing [3], resectability increasing [4], elimination of micrometastases [5], and improving survival of patients $[6,7]$.

The evaluation of the efficacy of NCT is becoming increasingly important. Pathologically, except for TNM stage, tumor regression grade (TRG) is widely used to assess the reaction of tumor [8]. 
There are many different TRG standards, such as standards comparing the relative relationship between residual tumor and fibrosis [9], standards calculating the proportion of residual tumor in the tumor bed [10], and standards only pay attention to the amount of residual tumor [11]. Nevertheless, most studies showed TRG could be used as a predictor for prognosis, especially when grouped into responders and non-responders $[12,13]$.

TRG plays an important role in the evaluation of effectiveness of neoadjuvant therapy. TRG is related to many pathological characteristics, including ypT [14], ypN [15], histological type [16] and, Lauren classification [17]. However, the relationships between TRG and some clinical factors such as tumor markers before treatment are still unclear and it is unknown which groups of patients tend to have a better tumor regression.

In this study, we verified the prognostic significance of TRG, investigated the associations between TRG and some clinical factors, and explored the predictors of TRG.

\section{Methods}

\section{Patients}

The information about patients who had locally advanced gastric adenocarcinoma and received NCT between January 2010 and July 2016 at our institute were identified from our electronic database. The criteria for inclusion were: (1) pathologically proved gastric adenocarcinoma; (2) locally advanced gastric cancer (8th American Joint Committee on Cancer [AJCC] clinical stage II-III); (3) received NCT with or without postoperative treatment; and (4) underwent curative gastrectomy surgery. The exclusion criteria were: (1) received preoperative radiotherapy; (2) gastric remnant cancer or suffering from other malignant tumors; or (3) incomplete information on staging or tumor marker before treatment. Among 3,196 patients, 290 were locally advanced gastric cancer patients who underwent neoadjuvant therapy. 141 had all three serum tumor markers before neoadjuvant therapy and 95 had all three serum tumor markers before and after neoadjuvant therapy.

\section{Pathological response assessment}

The slices or blocks indicating the primary tumor of all patients were retrieved from the biospecimen library of our hospital. Two experienced gastrointestinal pathologists (Y.Z. and D.L.) reviewed all slices respectively without the knowledge of clinicopathological information of patients. Pathological TNM stage was reevaluated in accordance with the eighth edition of the AJCC cancer staging guideline. Pathological response of the primary tumor was assessed according to the CAP system: TRG 0 (No viable cancer cells, i.e. complete response), TRG 1 (Single cells or rare small groups of cancer cells, i.e. near complete response), TRG 2 (Residual cancer with evident tumor regression, but more than single cells or rare small groups of cancer cells, i.e. partial response), and TRG 3 (Extensive residual cancer with no evident tumor regression, i.e. poor or no response). When there was disagreement between pathologists, a consensus would be reached by joint rereview and discussion through a multihead microscope. Other extracted clinicopathological characteristics were reconfirmed during the evaluation process.

\section{Measurement of serum tumor markers}

The levels of serum tumor markers before neoadjuvant chemotherapy (pre-) were measured within 14 days before initial treatment, and the levels after neoadjuvant (post-) chemotherapy were measured within 14 days before the gastrectomy. The changes of tumor markers (diff-) indicated differences between post- and pre- groups. The cutoff values of CA199, CA125 and CA724 were $37 \mathrm{U} / \mathrm{ml}, 35 \mathrm{U} / \mathrm{ml}$ and $8.2 \mathrm{U} / \mathrm{ml}$, respectively.

\section{Statistical methods}

The relationships of categorical variables were calculated by the chi-square test or Fisher's exact test. Logistic regression analysis was used to identify the factors associated with pathological response. To explore the predictors of the pathological response, only clinical variables were included in the analysis. Cox proportional hazard regression model was used to assess the prognostic risk of clinical variables. Variables with $p<0.05$ in the multivariate analysis were considered significant. Survival curves were obtained using the Kaplan-Meier method, and log-rank test was used to compare survival differences. All patients were followed up every three months during the first two years, every six months for the following three years and annually thereafter. Overall survival (OS) was defined as the time from the first day of neoadjuvant therapy to the day of death from any cause or last follow-up day. Data was processed by SPSS ver. 25.0 (IBM Corp., Armonk, NY) and R 3.6.1 software (R Foundation for Statistical Computing, Vienna, Austria).

\section{Results}

\section{Patient characteristics}

The clinical features of 141 patients are shown in Table 1, and the pathological characteristics are shown in Table 2. There were 97 males $(68.8 \%)$ and 44 females $(31.2 \%)$, with age ranging from 33 to 76 years 
(median 58 years). Most of the tumors were located in the lower third part of the stomach $(57.4 \%)$, and only 2 $(1.4 \%)$ were located in the gastroesophageal junction (GEJ). Most patients underwent preoperative chemotherapy with SOX $(79.4 \%)$, and few patients received FOLFOX (17.0\%) and XELOX (3.5\%). The median number of NCT cycle was 2 (range from 2 to 4 ). The median operation interval, the time between the completion of neoadjuvant treatment and surgery, was 32 days, with an interquartile range from 29 to 37 days. The median follow-up time of all patients was 36 months (range from 3 to 81 months).

Table 1. Patients' characteristics according to CAP TRG

\begin{tabular}{|c|c|c|c|c|c|}
\hline Variable & $\begin{array}{l}\text { TRG 0-1 } \\
(\mathrm{n}=40)\end{array}$ & $\begin{array}{l}\text { TRG } 2 \\
(n=35)\end{array}$ & $\begin{array}{l}\text { TRG } 3 \\
(n=66)\end{array}$ & $\mathrm{P}$ & No. (\%) \\
\hline \multicolumn{4}{|l|}{ Gender } & \multicolumn{2}{|l|}{0.308} \\
\hline Male & $24(24.7)$ & $24(24.7)$ & $49(50.5)$ & & $97(68.8)$ \\
\hline Female & $16(36.4)$ & $11(25.0)$ & $17(38.6)$ & & $44(31.2)$ \\
\hline \multicolumn{4}{|l|}{ Age (yr) } & \multicolumn{2}{|l|}{0.555} \\
\hline$<65$ & $32(29.1)$ & $25(22.7)$ & $53(48.2)$ & & $110(78.0)$ \\
\hline$\geq 65$ & $8(25.8)$ & $10(32.3)$ & $13(41.9)$ & & $31(22.0)$ \\
\hline \multicolumn{4}{|c|}{ Tumor location } & \multicolumn{2}{|l|}{0.074} \\
\hline UGEJ & $4(19.4)$ & $7(33.3)$ & $10(47.6)$ & & $21(14.9)$ \\
\hline Middle third & $13(54.2)$ & $5(20.8)$ & $6(25.0)$ & & $24(17.0)$ \\
\hline Lower third & $18(22.2)$ & $20(24.7)$ & $43(53.1)$ & & $81(57.4)$ \\
\hline Diffuse & $5(33.3)$ & $3(20.0)$ & $7(46.7)$ & & 15 (10.6) \\
\hline \multicolumn{4}{|c|}{ Tumor size (cm) } & \multicolumn{2}{|c|}{0.012} \\
\hline$<5$ & $19(38.0)$ & $16(32.0)$ & $15(30.0)$ & & $50(35.5)$ \\
\hline$\geq 5$ & $21(23.1)$ & $19(20.9)$ & $51(56.0)$ & & $91(64.5)$ \\
\hline \multicolumn{4}{|l|}{ cT } & \multicolumn{2}{|l|}{0.504} \\
\hline $2-3$ & $2(20.0)$ & $4(40.0)$ & $4(40.0)$ & & $10(7.1)$ \\
\hline 4 & $38(29.0)$ & $31(23.7)$ & $62(47.3)$ & & $131(92.9)$ \\
\hline \multicolumn{4}{|l|}{ cN } & \multicolumn{2}{|l|}{0.282} \\
\hline- & $16(33.3)$ & $14(29.2)$ & 18 (37.5) & & $48(34.0)$ \\
\hline+ & $24(25.8)$ & $21(22.6)$ & 48 (51.6) & & $93(66.0)$ \\
\hline \multicolumn{4}{|l|}{ NCT } & \multicolumn{2}{|l|}{0.101} \\
\hline FOLFOX & $4(16.7)$ & $3(12.5)$ & $17(70.8)$ & & $24(17.0)$ \\
\hline SOX & $34(30.4)$ & $30(26.8)$ & $48(42.9)$ & & $112(79.4)$ \\
\hline XELOX & $2(40.0)$ & $2(40.0)$ & $1(20.0)$ & & $5(3.5)$ \\
\hline \multicolumn{3}{|l|}{ NCT cycles } & & \multicolumn{2}{|l|}{0.846} \\
\hline 2 & $22(25.9)$ & $22(25.9)$ & 41 (48.2) & & $85(60.3)$ \\
\hline 3 & 8 (38.1) & $5(23.8)$ & 8 (38.1) & & $21(14.9)$ \\
\hline 4 & $10(28.6)$ & $8(22.9)$ & 17 (48.6) & & $35(24.8)$ \\
\hline \multicolumn{3}{|l|}{ pre-CA199 } & & \multicolumn{2}{|l|}{0.377} \\
\hline - & $36(30.8)$ & $28(23.9)$ & $53(45.3)$ & & $117(83.0)$ \\
\hline+ & 4 (16.7) & $7(29.2)$ & $13(54.2)$ & & $24(17.0)$ \\
\hline \multicolumn{4}{|l|}{ pre-CA125 } & \multicolumn{2}{|l|}{0.856} \\
\hline- & $38(28.8)$ & $33(25.0)$ & $61(46.2)$ & & 132 (93.6) \\
\hline+ & $2(22.2)$ & $2(22.2)$ & $5(55.6)$ & & $9(6.4)$ \\
\hline \multicolumn{4}{|l|}{ pre-CA724 } & \multicolumn{2}{|l|}{0.047} \\
\hline- & $36(33.6)$ & $25(23.4)$ & $46(43.0)$ & & 107 (75.9) \\
\hline+ & $4(11.8)$ & $10(29.4)$ & $20(58.8)$ & & $34(24.1)$ \\
\hline
\end{tabular}

Note: TRG, tumor regression grade; UGEJ, upper third and gastroesophageal junction.

\section{Pathological assessment}

The examples of CAP TRG are shown in Fig. 1. Totally, 693 slices indicating surgical specimens were reviewed. The median number of reviewed slices was 4 , with an interquartile range from 3 to 5 . After revaluation, the number of patients was $4,36,35$ and
66 in the group of TRG 0-3, respectively. There was no significant difference in survival between TRG 0 and TRG 1 ( $\mathrm{P}=0.775)$, so these two categories were classified into the responder group. Similarly, no significant difference was found between TRG 2 and TRG 3 ( $\mathrm{P}=0.383)$, so these two categories were classified into the non-responder group. The survival curves of CAP TRG were shown in Fig. 2.

Table 2. Patients' characteristics according to CAP TRG

\begin{tabular}{|c|c|c|c|c|c|}
\hline Variable & $\begin{array}{l}\text { TRG 0-1 } \\
(\mathrm{n}=40)\end{array}$ & $\begin{array}{l}\text { TRG } 2 \\
(\mathrm{n}=35)\end{array}$ & $\begin{array}{l}\text { TRG } 3 \\
(\mathrm{n}=66)\end{array}$ & $\mathrm{P}$ & No. $(\%)$ \\
\hline \multicolumn{3}{|l|}{ ypT } & \multicolumn{3}{|c|}{$<0.001$} \\
\hline 0 & $4(100.0)$ & $0(0.0)$ & $0(0.0)$ & & $4(2.6)$ \\
\hline $1-2$ & $20(87.0)$ & $1(4.3)$ & $2(8.7)$ & & $23(16.3)$ \\
\hline $3-4$ & $16(14.0)$ & $34(29.8)$ & $64(56.1)$ & & $114(80.9)$ \\
\hline \multicolumn{3}{|l|}{ ypN } & \multicolumn{3}{|c|}{$<0.001$} \\
\hline 0 & $23(53.5)$ & $9(20.9)$ & $11(25.6)$ & & $43(30.5)$ \\
\hline 1 & $9(32.1)$ & $8(28.6)$ & $11(39.3)$ & & $28(19.9)$ \\
\hline 2 & $6(15.4)$ & $9(23.1)$ & $24(61.5)$ & & $39(27.7)$ \\
\hline 3 & $2(6.5)$ & $9(29.0)$ & $20(64.5)$ & & $31(22.0)$ \\
\hline \multicolumn{3}{|l|}{ ypTNM } & \multicolumn{3}{|c|}{$<0.001$} \\
\hline $1-2$ & $32(59.3)$ & $10(18.5)$ & $12(22.2)$ & & $54(38.3)$ \\
\hline 3 & $8(9.2)$ & $25(28.7)$ & $54(62.1)$ & & $87(61.7)$ \\
\hline \multicolumn{3}{|l|}{ Histological type } & \multicolumn{3}{|c|}{$<0.001$} \\
\hline Adenocarcinoma & $34(38.6)$ & $24(27.3)$ & $30(34.1)$ & & $88(62.4)$ \\
\hline Poorly cohesive carcinoma & $6(11.3)$ & $11(20.8)$ & $36(67.9)$ & & $53(37.6)$ \\
\hline \multicolumn{3}{|l|}{ Lauren classification } & \multicolumn{3}{|c|}{0.037} \\
\hline Intestinal & $27(38.0)$ & $15(21.1)$ & $29(40.8)$ & & $71(50.4)$ \\
\hline Diffuse or mixed & $13(18.6)$ & $20(28.6)$ & $37(52.9)$ & & $70(49.6)$ \\
\hline \multicolumn{3}{|l|}{ Grade of differentiation } & \multicolumn{3}{|c|}{$<0.001$} \\
\hline Well & $21(58.3)$ & $5(13.9)$ & $10(27.8)$ & & $36(25.5)$ \\
\hline Moderate or poor & $19(18.1)$ & $30(28.6)$ & $56(53.3)$ & & $105(74.5)$ \\
\hline \multicolumn{3}{|c|}{ Vascular or lymphatic invasion } & \multicolumn{3}{|c|}{0.019} \\
\hline No & $35(35.0)$ & $24(24.0)$ & $41(41.0)$ & & $100(70.9)$ \\
\hline Yes & $5(12.2)$ & $11(26.8)$ & $25(61.0)$ & & $41(29.1)$ \\
\hline \multicolumn{3}{|l|}{ Nervous invasion } & \multicolumn{3}{|c|}{$<0.001$} \\
\hline No & $40(39.2)$ & $18(17.6)$ & $44(43.1)$ & & $102(72.3)$ \\
\hline Yes & $0(0.0)$ & $17(43.6)$ & $22(56.4)$ & & $39(27.7)$ \\
\hline \multicolumn{3}{|l|}{ Adjuvant treatment } & \multicolumn{3}{|c|}{0.319} \\
\hline No & $8(42.1)$ & $3(15.8)$ & $9(47.4)$ & & $19(13.5)$ \\
\hline Yes & $32(26.2)$ & $32(26.2)$ & $58(47.5)$ & & $122(86.5)$ \\
\hline
\end{tabular}

For tumor markers, all included patients have information on three tumor markers before treatment. The numbers of patients who had positive tumor markers were 24, 9 and 34 for pre-CA199, pre-CA125 and pre-CA724, respectively. Patients with high level of pre-CA724 tended to have a worse pathological response $(P=0.047)$. However, no similar associations were found for pre-CA199 $(\mathrm{P}=0.377)$ and pre-CA125 $(\mathrm{P}=0.856)$ (Table 1). The univariable analysis showed that high level of pre-CA724 was associated with poor pathological response $(\mathrm{OR}=3.803, \mathrm{P}=0.019)$, while the pre-CA199 $(\mathrm{P}=0.171)$ and pre-CA125 $(\mathrm{P}=0.674)$ were not relevant to the tumor reaction (Table 3). In multivariable analysis, pre-CA724 was an 
independent risk factor for the pathological response $(\mathrm{P}=0.032)$ (Table 3 ). In addition, 95 patients who also had information on tumor markers after neoadjuvant therapy were analyzed, but CA724 was no longer an independent risk factor $(\mathrm{P}=0.150)$ (data not shown).

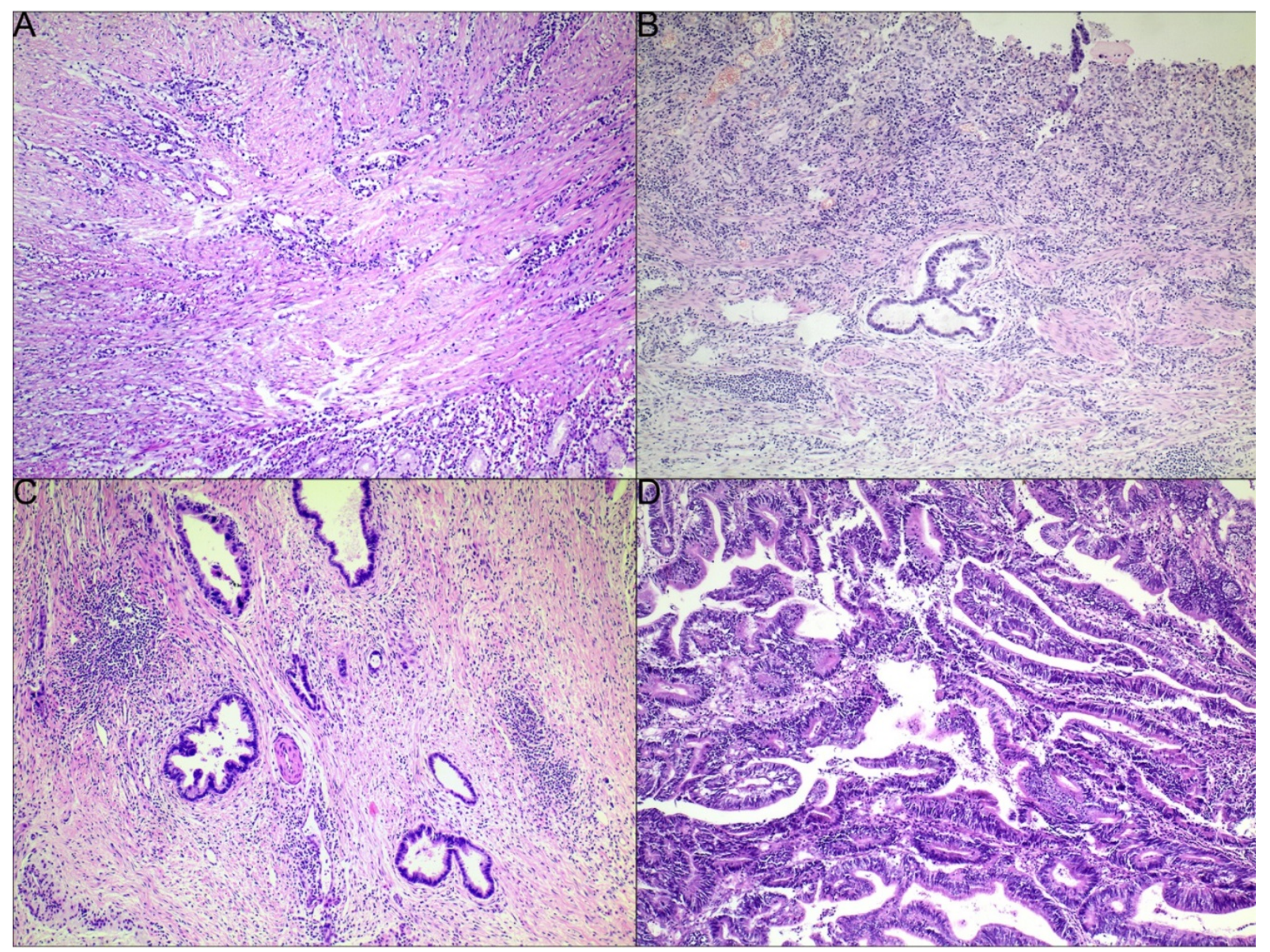

Figure 1. Examples of CAP TRG (A-D): (A) TRG 0, complete tumor regression; (B) TRG 1, single cells or rare small groups of cancer cells; (C) TRG 2, residual cancer with evident tumor regression, but more than single cells or rare small groups of cancer cells; (D) TRG 3, extensive residual cancer with no evident tumor regression.
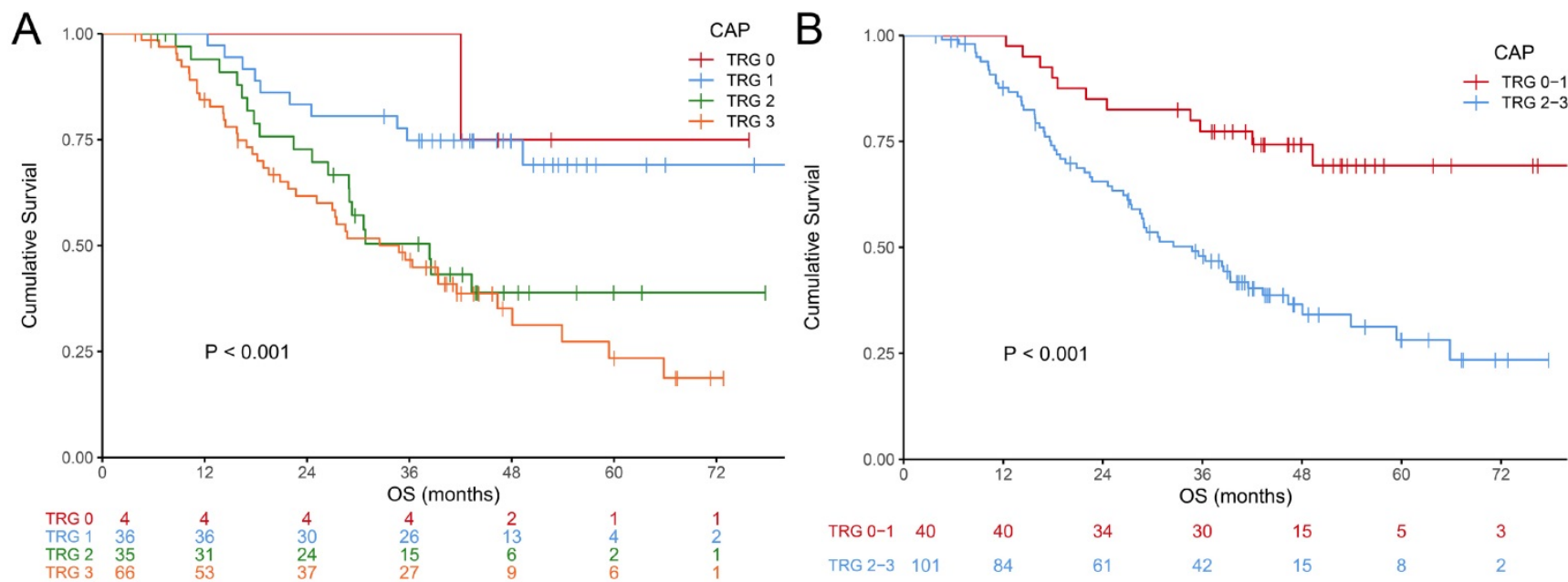

Figure 2. Kaplan-Meier curves for overall survival of CAP TRG. (A) four-tier TRG; (B) pathological response (TRG 0-1 vs 2-3). 

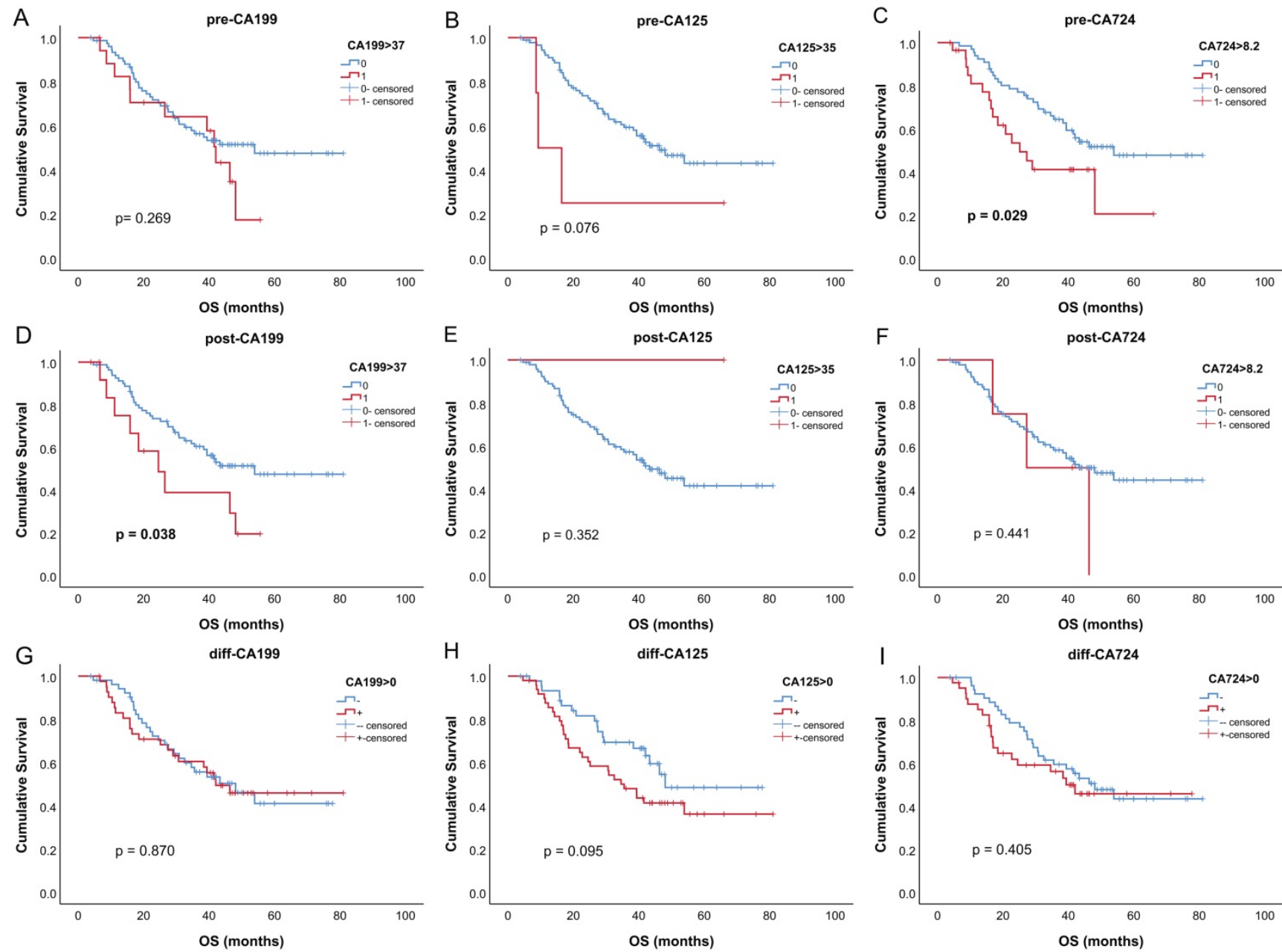

Figure 3. Kaplan-Meier curves for overall survival of tumor markers. (A-C) before neoadjuvant chemotherapy (NCT); (D-F) after NCT; (G-I) change between before and after NCT.

Table 3. Logistic analysis for pathological response

\begin{tabular}{|c|c|c|c|c|}
\hline \multirow[t]{2}{*}{ Variable } & \multicolumn{2}{|l|}{ Univariable analysis } & \multicolumn{2}{|c|}{ Multivariable analysis } \\
\hline & OR $(95 \% \mathrm{CI})$ & $\mathrm{P}$ & OR $(95 \% \mathrm{CI})$ & $\mathrm{P}$ \\
\hline Gender (Female) & $0.575(0.267,1.240)$ & 0.158 & $0.618(0.241,1.583)$ & 0.316 \\
\hline Age ( $\geq 65 \mathrm{yr})$ & $1.179(0.478,2.912)$ & 0.555 & $0.991(0.338,2.902)$ & 0.987 \\
\hline Tumor location & & 0.022 & & 0.047 \\
\hline UGEJ & 1 & & 1 & \\
\hline Middle third & $0.199(0.051,0.770)$ & 0.019 & $0.132(0.028,0.615)$ & 0.010 \\
\hline Lower third & $0.824(0.246,2.758)$ & 0.753 & $0.477(0.125,1.821)$ & 0.279 \\
\hline Diffuse & $0.471(0.102,2.172)$ & 0.334 & $0.195(0.034,1.125)$ & 0.068 \\
\hline Tumor size $(\geq 5 \mathrm{~cm})$ & $2.043(0.964,4.329)$ & 0.062 & $3.433(1.323,8.913)$ & 0.011 \\
\hline cT (4) & $0.612(0.124,3.015)$ & 0.546 & $0.467(0.075,2.919)$ & 0.416 \\
\hline $\mathrm{cN}(+)$ & $1.437(0.673,3.070)$ & 0.349 & $1.413(0.578,3.454)$ & 0.449 \\
\hline NCT & & 0.352 & & 0.455 \\
\hline FOLFOX & 1 & & 1 & \\
\hline SOX & $0.459(0.146,1.444)$ & 0.183 & $0.545(0.149,1.994)$ & 0.359 \\
\hline XELOX & $0.300(0.037,2.417)$ & 0.258 & $0.244(0.024,2.498)$ & 0.235 \\
\hline NCT cycles & & 0.543 & & 0.737 \\
\hline 2 & 1 & & 1 & \\
\hline 3 & $0.567(0.208,1.551)$ & 0.269 & $0.654(0.189,2.257)$ & 0.501 \\
\hline 4 & $0.873(0.362,2.104)$ & 0.762 & $0.739(0.259,2.103)$ & 0.570 \\
\hline CA199 (+) & $2.222(0.709,6.970)$ & 0.171 & $1.533(0.431,5.449)$ & 0.509 \\
\hline CA125 (+) & $1.415(0.281,7.121)$ & 0.674 & $1.059(0.157,7.148)$ & 0.953 \\
\hline CA724 (+) & $3.803(1.244,11.628)$ & 0.019 & $4.033(1.128,14.427)$ & 0.032 \\
\hline
\end{tabular}

Note: UGEJ, upper third and gastroesophageal junction.
For other characteristics, tumor size was associated with TRG. Patients with larger tumor tended to have a worse TRG $(\mathrm{P}=0.012)$ (Table 1). In logistic analysis, tumor size did not contribute to a worse pathological response in univariable analysis $(\mathrm{P}$ $=0.062)$, but in multivariable analysis, it became an independent risk factor $(\mathrm{P}=0.011)$ (Table 3). It was surprising that tumor location was related to pathological response in the logistic analysis, and tumor in the middle third of the stomach tended to have a better pathological response when compared with tumor located in the gastroesophageal junction and the upper third of the stomach $(\mathrm{P}<0.05)$.

\section{Prognostic values of serum tumor markers}

For patients who had all tumor markers before and after neoadjuvant therapy, survival analysis was performed. Survival curves of all tumor markers were shown in Fig. 3. Only pre-CA724 ( $p=0.029)$ and postCA199 ( $p=0.038$ ) were related to overall survival. In univariable analysis, pre-CA724 was related to prognosis $(p=0.032)$, while pre-CA199 $(p=0.272)$ and 
pre-CA125 ( $p=0.089$ ) were not. In multivariable analysis, pre-CA724 was an independent prognostic factor $(p=0.015)$ (Table 4). However, in multivariable analysis including tumor markers after neoadjuvant therapy, post-CA199 $(p=0.007)$ was an independent prognostic factor while post-CA724 $(\mathrm{p}=0.723)$ was not (Table S1). In multivariable analysis including changes of tumor markers, none of the three tumor markers was related to overall survival (all $p>0.05$ ) (data not shown).

Table 4. Cox proportional hazard regression model for overall survival before neoadjuvant therapy

\begin{tabular}{|c|c|c|c|c|}
\hline \multirow[t]{2}{*}{ Variable } & \multicolumn{2}{|l|}{ Univariable analysis } & \multicolumn{2}{|l|}{ Multivariable analysis } \\
\hline & OR $(95 \% \mathrm{CI})$ & $\mathrm{P}$ & OR $(95 \% \mathrm{CI})$ & $\mathrm{P}$ \\
\hline Gender (Female) & $1.460(0.757,2.817)$ & 0.259 & $0.734(0.364,1.480)$ & 0.38 \\
\hline Age ( $\geq 65 \mathrm{yr})$ & $1.268(0.706,2.275)$ & 0.427 & $1.012(0.481,2.130)$ & 0.976 \\
\hline Tumor location & & 0.037 & & 0.008 \\
\hline Middle third & 1 & & 1 & \\
\hline UGEJ & $2.030(0.523,7.874)$ & 0.306 & $2.366(0.545,10.270)$ & 0.250 \\
\hline Lower third & $2.161(0.654,7.141)$ & 0.206 & $4.263(0.932,19.494)$ & 0.062 \\
\hline Diffuse & $5.186(1.415,19.012)$ & 0.013 & $9.427(2.161,41.125)$ & 0.003 \\
\hline Tumor size $(\geq 5 \mathrm{~cm})$ & $2.162(1.137,4.110)$ & 0.019 & $2.307(1.093,4.871)$ & 0.028 \\
\hline cT (4) & $0.318(0.123,0.817)$ & 0.017 & $0.300(0.098,0.918)$ & 0.035 \\
\hline $\mathrm{cN}(+)$ & $1.494(0.809,2.760)$ & 0.200 & $1.472(0.733,2.957)$ & 0.277 \\
\hline NCT & & 0.589 & & 0.069 \\
\hline FOLFOX & 1 & & 1 & \\
\hline SOX & $1.628(0.644,4.117)$ & 0.303 & $3.642(1.218,10.890)$ & 0.021 \\
\hline XELOX & * & 0.979 & * & ** \\
\hline NCT cycles & & 0.642 & & 0.008 \\
\hline 2 & 1 & & 1 & \\
\hline 3 & $0.900(0.437,1.855)$ & 0.775 & $1.309(0.531,3.228)$ & 0.559 \\
\hline 4 & $0.697(0.329,1.479)$ & 0.347 & $0.283(0.110,0.724)$ & 0.008 \\
\hline pre-CA199 (+) & $1.461(0.743,2.875)$ & 0.272 & $2.011(0.887,4.563)$ & 0.095 \\
\hline pre-CA125 (+) & $2.776(0.856,9.004)$ & 0.089 & $6.012(1.429,25.284)$ & 0.014 \\
\hline pre-CA724 (+) & $1.949(1.059,3.586)$ & 0.032 & $2.935(1.232,6.991)$ & 0.015 \\
\hline
\end{tabular}
large to record.

\section{Discussion}

As neoadjuvant therapy has been successfully introduced in the gastrointestinal malignancy, the assessment of efficacy of preoperative therapy is of great importance. Tumor regression grade is a widely used standard to evaluate the effectiveness, but there are many different versions. This study is based on CAP TRG, which is derived from Mandard TRG [9], but a little different [8]. Mandard TRG focuses on the relative relationship between residual tumor and fibrosis while CAP TRG merely concentrates on the amount of residual tumor. Compared with Mandard TRG, CAP TRG does not need to distinguish between fibrosis due to tumor regression and fibrosis in the normal area that has never been invaded by tumor. In other words, CAP TRG is easier to carry out and has a better consistency among observers. Nevertheless, this standard still needs to be improved to clearly distinguish different grades by using specific quantities of residual tumor. Although there are some differences between these two TRG standards, these differences will be narrowed when they are divided into responders and non-responders, based on their definitions.

Many researches have proved that responders have a better prognosis than non-responders, so if relationships between TRG and other clinical factors could be revealed, it is possible to predict TRG. Therefore, this study explored the predictors of TRG from characteristics before treatment, and found that CA724 before treatment was an independent risk factor to pathological reaction. In this aspect, only a few researches concentrated on the association of tumor markers and neoadjuvant therapy in gastric cancer. Sun et al. [18] found that CA199 and CA724 were related to overall survival in patients who underwent neoadjuvant chemotherapy, but these two tumor markers were not related to pathological response. This difference might be because their study was based on Backer TRG, and they used a different cutoff value on CA724. Zou et al. [19] found that the decline of CA724 was related to the effectiveness of neoadjuvant therapy. The difference was that they used RECIST criteria rather than TRG. In our another article [20], we found that CA724 before treatment and CA724 after treatment were both independent predictive factors for overall survival. However, in that article, TRG was not mentioned. In addition, tumor size and tumor location showed the predictive power to TRG. However, because of lack of evidence, more researches based on larger sample size are needed to verify this result.

This study also found TRG was related to various pathological factors. This conclusion is similar to other studies. In other studies, TRG was found to be associated with ypT stage [14, 15, 21], ypN stage [22, 23], histological type [16], Lauren classification [17], differentiation grade [15, 23], lymphovascular invasion [21, 23] and nervous invasion [24]. Nevertheless, these characteristics after treatment could not be used as a predictor to TRG because these factors and TRG are all obtained at the stage of pathological evaluation.

There are some limitations in this study. This study is retrospective and conducted at a single institution, which means there might be a potential selection bias. This study is based on a relatively small sample size, so a subgroup analysis was not performed. This study used normal cutoff values of tumor markers; however, it is unknown that whether the optimal cutoff values would change because of preoperative treatment. To determine the optimal cutoff values of tumor markers, further researches based on larger sample size are needed. Nevertheless, our study was based on a specific group of patients, explored predictors of TRG from clinical 
characteristics, and found a relationship between tumor markers and TRG.

In conclusion, CA724 before NCT was an independent prognostic factor for prognosis and could be used as a predictor for TRG in locally advanced gastric cancer patients who underwent neoadjuvant therapy and curative operation.

\section{Abbreviations}

GC: gastric cancer; NCT: neoadjuvant chemotherapy; TRG: tumor regression grade; OS: overall survival; AJCC: American Joint Committee on Cancer; CA199: carbohydrate antigen 19-9; CA125: cancer antigen 125; CA724: carbohydrate antigen 72-4; GEJ: gastroesophageal junction; ypTNM: post-neoadjuvant therapy tumor-node-metastasis stage.

\section{Supplementary Material}

Supplementary table.

http://www.jcancer.org/v12p6465s1.pdf

\section{Acknowledgments}

This study was supported by grants from Natural Science Foundation of Liaoning Province of China (2020-MS-057), Key Research and Development Project of Liaoning Province (2020JH2/10300043), and Shenyang High-level Innovative Talents Program (RC190447).

\section{Ethics Committee Approval and Patient Consent}

The study was reviewed and approved by the Faculty of Science Ethics Committee at Liaoning Cancer Hospital and Institute (Cancer Hospital of China Medical University). This is a retrospective study and all patients have signed the informed consent prior to treatment.

\section{Author Contributions}

YT performed the majority of experiments and analyzed the data and drafted the manuscript; YMZ and DL reevaluated pathological data; YZ reviewed and revised the manuscript; CJ, WW, ZS and FS assisted in collected and analyzed the data; JZ supervised the study and provided critical revision of the manuscript. All authors have read and approved the manuscript.

\section{Competing Interests}

The authors have declared that no competing interest exists.

\section{References}

1. Siegel RL, Miller KD, Jemal A. Cancer statistics, 2020. CA Cancer J Clin. 2020; 70: 7-30.
2. Davies AR, Gossage JA, Zylstra J, Mattsson F, Lagergren J, Maisey N, et al. Tumor stage after neoadjuvant chemotherapy determines survival after surgery for adenocarcinoma of the esophagus and esophagogastric junction. J Clin Oncol. 2014; 32: 2983-90.

3. Martin-Romano P, Sola JJ, Diaz-Gonzalez JA, Chopitea A, Iragorri Y, Martinez-Regueira F, et al. Role of histological regression grade after two neoadjuvant approaches with or without radiotherapy in locally advanced gastric cancer. Br J Cancer. 2016; 115: 655-63.

4. Smyth EC, Fassan M, Cunningham D, Allum WH, Okines AF, Lampis A, et al. Effect of Pathologic Tumor Response and Nodal Status on Survival in the Medical Research Council Adjuvant Gastric Infusional Chemotherapy Trial. J Clin Oncol. 2016; 34: 2721-7.

5. Fokas E, Liersch T, Fietkau R, Hohenberger W, Beissbarth T, Hess C, et al. Tumor regression grading after preoperative chemoradiotherapy for locally advanced rectal carcinoma revisited: updated results of the CAO/ARO/AIO-94 trial. J Clin Oncol. 2014; 32: 1554-62.

6. Al-Batran SE, Homann N, Pauligk C, Goetze TO, Meiler J, Kasper S, et al. Perioperative chemotherapy with fluorouracil plus leucovorin, oxaliplatin, and docetaxel versus fluorouracil or capecitabine plus cisplatin and epirubicin for locally advanced, resectable gastric or gastro-oesophageal junction adenocarcinoma (FLOT4): a randomised, phase 2/3 trial. Lancet. 2019; 393: 1948-57.

7. Al-Batran SE, Homann N, Pauligk C, Illerhaus G, Martens UM, Stoehlmacher J, et al. Effect of Neoadjuvant Chemotherapy Followed by Surgical Resection on Survival in Patients With Limited Metastatic Gastric or Gastroesophageal Junction Cancer: The AIO-FLOT3 Trial. JAMA Oncol. 2017; 3: 1237-44.

8. Tong Y, Liu D, Zhang J. Connection and distinction of tumor regression grading systems of gastrointestinal cancer. Pathol Res Pract. 2020; 216: 153073.

9. Mandard AM, Dalibard F, Mandard JC, Marnay J, Henry-Amar M, Petiot JF, et al. Pathologic assessment of tumor regression after preoperative chemoradiotherapy of esophageal carcinoma. Clinicopathologic correlations. Cancer. 1994; 73: 2680-6.

10. Becker K, Mueller JD, Schulmacher C, Ott K, Fink U, Busch R, et al. Histomorphology and grading of regression in gastric carcinoma treated with neoadjuvant chemotherapy. Cancer. 2003; 98: 1521-30.

11. Chanjuan Shi, Jordan Berlin, Philip A. Branton, Patrick L. Fitzgibbons, Wendy L. Frankel, Wayne L. Hofstetter, et al. Protocol for the Examination of Specimens From Patients With Carcinoma of the Stomach (Stomach 4.1.0.0) 2020: 5-6.

12. Tomasello G, Petrelli F, Ghidini M, Pezzica E, Passalacqua R, Steccanella $\mathrm{F}$, et al. Tumor regression grade and survival after neoadjuvant treatment in gastro-esophageal cancer: A meta-analysis of 17 published studies. Eur J Surg Oncol. 2017; 43: 1607-16.

13. Kong JC, Guerra GR, Warrier SK, Lynch AC, Michael M, Ngan SY, et al. Prognostic value of tumour regression grade in locally advanced rectal cancer: a systematic review and meta-analysis. Colorectal Dis. 2018; 20: 574-85.

14. Fokas E, Strobel P, Fietkau R, Ghadimi M, Liersch T, Grabenbauer GG, et al. Tumor Regression Grading After Preoperative Chemoradiotherapy as a Prognostic Factor and Individual-Level Surrogate for Disease-Free Survival in Rectal Cancer. J Natl Cancer Inst. 2017; 109(12).

15. Becker K, Langer R, Reim D, Novotny A, Meyer zum Buschenfelde C, Engel J, et al. Significance of histopathological tumor regression after neoadjuvant chemotherapy in gastric adenocarcinomas: a summary of 480 cases. Ann Surg. 2011; 253: 934-9.

16. Puetz K, Bollschweiler E, Semrau R, Monig SP, Holscher AH, Drebber U. Neoadjuvant chemoradiation for patients with advanced esophageal cancer- Which response grading system best impacts prognostic discrimination? Histopathology. 2019; 74: 731-43.

17. van der Kaaij RT, Snaebjornsson P, Voncken FE, van Dieren JM, Jansen EP, Sikorska K, et al. The prognostic and potentially predictive value of the Lauren classification in oesophageal adenocarcinoma. Eur J Cancer. 2017; 76: 27-35.

18. Sun Z, Zhang N. Clinical evaluation of CEA, CA19-9, CA72-4 and CA125 in gastric cancer patients with neoadjuvant chemotherapy. World J Surg Oncol. 2014; 12: 397.

19. Zou L, Qian J. Decline of serum CA724 as a probable predictive factor for tumor response during chemotherapy of advanced gastric carcinoma. Chin J Cancer Res. 2014; 26: 404-9.

20. Tong Y, Zhao Y, Shan Z, Zhang J. CA724 predicts overall survival in locally advanced gastric cancer patients with neoadjuvant chemotherapy. BMC Cancer. 2021; 21: 4.

21. Rodel C, Martus P, Papadoupolos T, Fuzesi L, Klimpfinger M, Fietkau R, et al. Prognostic significance of tumor regression after preoperative chemoradiotherapy for rectal cancer. J Clin Oncol. 2005; 23: 8688-96.

22. Hagi T, Makino T, Yamasaki M, Yamashita K, Tanaka K, Saito T, et al. Pathological Regression of Lymph Nodes Better Predicts Long-term 
Survival in Esophageal Cancer Patients Undergoing Neoadjuvant Chemotherapy Followed by Surgery. Ann Surg. 2020 Jul 14.

23. Noble F, Lloyd MA, Turkington R, Griffiths E, O'Donovan M, O'Neill JR, et al. Multicentre cohort study to define and validate pathological assessment of response to neoadjuvant therapy in oesophagogastric adenocarcinoma. Br J Surg. 2017; 104: 1816-28.

24. Tong Y, Zhu Y, Zhao Y, Shan Z, Liu D, Zhang J. Evaluation and Comparison of Predictive Value of Tumor Regression Grades according to Mandard and Becker in Locally Advanced Gastric Adenocarcinoma. Cancer Res Treat. 2021; 53: 112-22. 